Journal of Al Azhar University Engineering Sector

Vol. 13, No. 49, October, 2018, 1240-1258

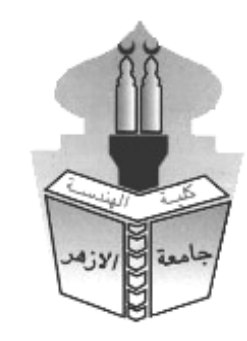

\title{
REVIEW ON ENHANCING TECHNOLOGIES OF HEAT TRANSFER THROUGH PHASE CHANGE MATERIALS
}

\author{
Mostafa Bahaa Ghonamy, Ehab Mouris and A. M. Abdul Aziz \\ Faculty of Engineering, Ain Shams university
}

\begin{abstract}
This review concentrates deeply on the recent studies performed on the phase change materials (PCMs) in order to enhance their performance. Most of these enhancing techniques fall in the following areas of interest (i) Development of new PCMs with promising thermo-physical properties. The researches recommend some promising substances to be used as a PCM such as pure inorganic salts (low cost chlorides, nitrates and carbonates), salt eutectics (KNO3-LiNO3 and KNO3-NaNO3-LiNO3), liquid metals (Gallium), esters, nanofluids (A12O3/water, $\mathrm{CuO} /$ water and alumina-water), and polyols (sugar alcohol like polyethelene glycol, erythritol and xylitol). (ii) Introducing of composite mixtures to exaggerate the benefits of used materials. Composites could be obtained by mixing PCMs like fatty acid esters with building materials like concrete pavements, cement

or gypsum or with isolating materials like perlite to keep satisfying room temperature for long time. PCMs could be mixed or be used in cascaded configuration layered and ordered according to their melting temperature. Good conducting materials like graphite and aluminum were introduced to be mixed with PCMs to enhance the thermal conductivity of PCM. Stable materials like Diatomite and silica were introduced to enhance the stability of the PCM to retain its properties for a lot of melting/freezing cycles. Also, nano particles of $\mathrm{TiO} 2, \mathrm{ZnO}, \mathrm{CuO}$, and Silver-Titania, are added to generally enhance the PCM thermo-physical properties. (iii) Configuration of PCM container to achieve higher heat transfer rates by changing the aspect ratio or by adding fins. The studies focused on adding internal fins, external fins, and increasing the aspect ratio of the PCM vertical container to induce natural convection. Experiments advise to focus on increasing the number and height of fins more than focusing of fins thickness. (iv) Encapsulation technology of PCM to provide the largest heat transfer surface and avoid leakage when in liquid state as well as allowing larger surface area for heat transfer and protection in handling hazardous materials. It was observed that the core-to-coating ratio plays an important role in deciding the thermal and structural stability of the encapsulated PCM. An increased coreto-coating ratio results in a weak encapsulation, whereas, the amount of PCM and hence the heat storage capacity decreases with a decreased core-to-coating ratio. Among all the microencapsulation methods, the most common methods described in the literature for the production of microencapsulated phase change materials (MEPCMs) are interfacial
\end{abstract}


polymerization, suspension polymerization, coacervation, emulsion polymerization, and spray drying.

\section{HIGHLIGHTS}

Techniques for enhancing the heat conductivity of phase change materials

Encapsulation methods for enhancing the performance of phase change materials.

Composite mixtures proposed for better thermal response of phase change materials

\section{INTRODUCTION}

There are numerous technologies for storing solar energy in various forms including mechanical, electrical and thermal energy. Thermal energy can be stored in well-insulated fluids or solids as a change in internal energy of a material as sensible heat, latent heat and thermo-chemical or combination of these. Phase change materials (PCMs) are used in latent thermal energy storage (LTES) system due to their large latent heats which allow maintaining constant temperature while storing thermal energy through a phase change process. The low thermal conductivity of PCMs which leads to long time required for phase change, also sometimes the PCM material lacks the stability which ensures same thermo-physical properties after repeated melting-solidification cycles. In last decades, the interest in using PCM for energy storage increased which encourages researchers to work on studying how to develop the thermal performance of PCMs. During studding the Phase change materials, it shows great promising results if better thermal response can be assured. During the ongoing decade, most of these enhancing techniques fall in the below areas of interest:

I- Study new PCMs with promising thermo-physical properties [1-8]

II- Introducing composite mixtures to allow better heat conductivity [9-31]

III- Configuration of Slab that contains the PCM [32-41]

IV- Encapsulation technology of PCM to allow better heat transfer [42-44]

\section{1- RESEARCHES ABOUT STUDYING NEW PCMS WITH PROMISING THERMO- PHYSICAL PROPERTIES}

Researchers worked on discovering and experimenting different materials to be introduced as promising PCMs with more efficient Thermo-physical properties. In 2012, M. Liu et al. [1] reviewed phase change materials with melting temperatures above $300{ }^{\circ} \mathrm{C}$. The promising substances include pure inorganic salts, salt eutectics, metals and metallic eutectic alloys. Most of the pure inorganic salts and salt eutectics included are on the basis of chlorides, nitrates and carbonates which have low cost. In 2013, K. Menoufi et al. [2] investigated the use of esters as PCM in order to estimate its environmental impact in building envelopes in comparison to the use of paraffin or salt hydrates through a theoretical study. It is found that the impact of ester used as PCM presents slightly better results than the case of using salt hydrates and paraffins. $\mathrm{H}$. Ge et al. [3] introduced low melting point liquid metals like Gallium as a new class of phase change material concluding that the metals and alloys with melting point between at $01 \mathrm{C}$ and $1001 \mathrm{C}$ are showing increasing potentials in various thermal and energy management fields, especially for those situations where the cooling space was strictly limited and the heat source works inconsecutively.

A. Gil et al. [4] studied the thermal behavior of "D-mannitol" when used as PCM. The experiments have shown that the D-mannitol presents structural changes and, therefore, its thermal properties are not always the same resulting in different melting temperature. F. Roget et al. [5] studied KNO3-LiNO3 and KNO3-NaNO3-LiNO3 eutectics as phase change materials for 
thermal storage in low temperatures, finding that the volume storage capacity of the LiNO3KNO3 eutectic was measured at $89 \mathrm{~kW} \mathrm{~h} / \mathrm{m}^{3}$ and that of the LiNO3-KNO3- NaNO3 eutectic at $86 \mathrm{~kW} \mathrm{~h} / \mathrm{m}^{3}$. In 2015, A. Sivakumar et al. [6] studied the heat transfer performance in nanofluids of $\mathrm{Al} 2 \mathrm{O} 3 /$ water and $\mathrm{CuO} /$ water resulting that there is enhanced heat transfer coefficient of $\mathrm{CuO} /$ water compared with $\mathrm{Al} 2 \mathrm{O} 3 /$ water showing also that there is increase in heat transfer coefficient of nanofluids $\mathrm{CuO}$ /water and $\mathrm{Al} 2 \mathrm{O} 3$ /water when compared with their base fluids. In 2016, M.M. MacDevette and T.G. Myers [7] studied if Nanofluids can be considered as innovative phase change material for cold storage systems by testing alumina-water nanofluid concluding that nanofluid based phase change materials may not provide the anticipated improvements in energy storage and release. S.N. Gunasekara et al. [8] introduced polyols (sugar alcohols) as a promising phase change materials for surplus thermal energy storage focusing on poyols in the range of 15 to $245 \mathrm{C}$, and considerable phase change enthalpies of $100-413 \mathrm{~kJ} / \mathrm{kg}$ like Polyethylene glycol, Erythritol, and Xylitol. Results indicated that polyols is generally an interesting material category even compared to other less expensive organics like paraffins and fatty acids.

\section{2- RESEARCHES ABOUT INTRODUCING COMPOSITE MIXTURES TO ALLOW BETTER HEAT CONDUCTIVITY}

Researchers worked on experimenting different material composites to help overcoming the drawbacks of regular PCMs with more efficient Thermo-physical properties. In 2011, A. Karaipekli and A. Sarı [9] study composites of fatty acid ester with building material for thermal energy storage in buildings. The composites of erythritol tetrapalmitate and erythritol tetrastearate esters were used as PCMs for thermal energy storage, and cement and gypsum used as supporting material.

Results showed that the melting temperatures and the latent heats of the prepared composite PCMs were in range of $21.6-32.3{ }^{\circ} \mathrm{C}$ and $35.9-43.3 \mathrm{~J} / \mathrm{g}$, respectively. The thermal cycling test including 1000 heating and cooling process was conducted to determine the thermal reliability of the composite PCMs and the test results revealed that the composite PCMs have good thermal reliability and chemical stability. Y. Wang et al. [10] proposed stearic acid/silica fume composite as form-stable phase change material for thermal energy storage. The latent heat of the composite PCM is measured as $82.53 \mathrm{~J} / \mathrm{g}$ for the melting process and $84.47 \mathrm{~J} / \mathrm{g}$ for the freezing process, respectively, which indicate the heat storage ability of composite is connected with the mass ratio of stearic acid in composite. The thermal cycling test showed that the thermal reliability of the composite PCM has an imperceptible change. The increase of thermal conductivity was also confirmed by comparing the melting time, freezing time and phase change time of the composite with that of stearic acid. All of the conclusions indicate that the composite has a better thermal conductivity and good thermal and chemical stability. In 2012, Y. Qian et al. [11] studied paraffin composites as thermal energy storage materials. Results showed that the paraffin composites had good thermal energy storage property as well as enhanced thermal stability. S.F. Hosseinizadeh et al. [12] investigated numerically the unconstrained melting of nano-enhanced phase change material. Their simulation results show that the nanoparticles cause an increase in thermal conductivity compared to conventional PCM. In his research, transient simulation of axisymmetric unconstrained melting inside a spherical shell was performed and validated.

In 2013, M. Mehrali et al. [13] prepared palmitic acid/graphene oxide composite as thermal energy storage materials. The temperatures of melting, freezing and latent heats of the composite measured were $60.45^{\circ} \mathrm{C}, 60.05{ }^{\circ} \mathrm{C}, 101.23 \mathrm{~kJ} / \mathrm{kg}$ and $101.49 \mathrm{~kJ} / \mathrm{kg}$, respectively. Thermal cycling 
test showed that the form-stable composite PCM has good thermal reliability and chemical stability. Thermal conductivity of the composite PCM was improved by more than three times from $0.21 \mathrm{w} / \mathrm{mk}$ to $1.02 \mathrm{w} / \mathrm{mk}$. As a result, due to their acceptable thermal properties, good thermal reliability, chemical stability and great thermal conductivities, the composite is recommended as highly conductive PCMs for thermal energy storage applications. S.-G. Jeong et al. [14] prepared PCM/diatomite composites by incorporating PCMs in the pores of diatomite to increase the form stability of PCMs and enhancing thermal properties. N-hexadecane, noctadecane and paraffin wax were used as PCMs, which have latent heat capacities of $254.7 \mathrm{~J} / \mathrm{g}$, $247.6 \mathrm{~J} / \mathrm{g}$ and $144.6 \mathrm{~J} / \mathrm{g}$, respectively, and melting points of $20.84{ }^{\circ} \mathrm{C}, 30.4{ }^{\circ} \mathrm{C}$ and $57.09{ }^{\circ} \mathrm{C}$, respectively. Results showed that the latent heat capacities of PCM/diatomite composites were $50 \%$ the value of pure PCMs, and that PCM/diatomite composites have greater thermal durability compared with pure PCM. X.L. Wang et al. [15] tested the capric and lauric acid mixtures with organic additives theoretically and experimentally for cold storage in high temperature cooling application. It was shown that the mixture at the presence of oleic acid offered an appropriate phase change temperature $\left(14.97{ }^{\circ} \mathrm{C}\right)$ and high latent heat of transition $(115.1 \mathrm{~kJ} / \mathrm{kg})$. The charging and discharging time of the cold storage ball were $340 \mathrm{~min}$ and $230 \mathrm{~min}$ with the charging and discharging capacity of $113,291 \mathrm{~kJ} / \mathrm{m} 3$ and $106,844 \mathrm{~kJ} / \mathrm{m} 3$, respectively.
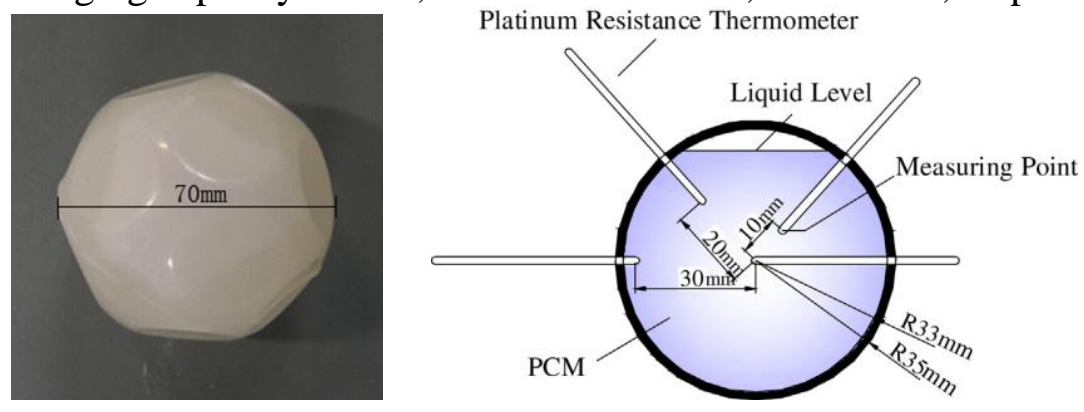

Figure (01): X.L. Wang study, Physical map of the cold storage ball and Sketch of the measuring points within the cold storage ball

The charging time can be reduced either by decreasing the heat transfer fluid temperature, the diameter and thickness of the spherical shell, or by increasing the thermal conductivity of the spherical shell. The cold storage ball manifests good suitability and stability for high temperature cooling application. M. Li [16] prepared nano-graphite (NG)/paraffin composites as composite phase change materials. The results indicated that the graphite layers were randomly dispersed in the paraffin, and the thermal conductivity increased gradually with the content of graphite. Thermal conductivity of the material containing $10 \%$ nano graphite were $0.9362 \mathrm{~W} / \mathrm{m} \mathrm{k}$ noting that the nano-graphite content increased, the thermal conductivity of the composite increased while the latent heat gradually decreased. In 2014, R. Parameshwaran et al.[17] experimented a new organic ester phase change material embedded with the silver-titania hybrid nanocomposite with the mass proportions ranging from $0.1 \%$ to $1.5 \%$ causing improvement of thermal conductivity from $0.286 \mathrm{~W} / \mathrm{m} \mathrm{K}$ to $0.538 \mathrm{~W} / \mathrm{m} \mathrm{K}$, high latent heat capacity $(90.81 \mathrm{~kJ} / \mathrm{kg})$, compared to the pure PCM. In total, the improved thermal properties and the heat storage potential of the composite has facilitated it to be considered as a viable candidate for the cool thermal energy storage applications in buildings without sacrificing energy efficiency. N. Zhang et al. [18] introduced Lauric-palmitic-stearic acid/expanded perlite composite as form-stable phase change material. The result of analysis revealed that the form-stable PCM had a good 
thermal stability. Moreover, the thermal conductivity was increased by $95 \%$ by adding $2 \%$ expanded graphite, and the thermal energy storage/release rates were also increased. Generally all results indicated that the prepared form-stable PCM composite can be considered as a potential material for building energy conservation due to satisfactory thermal properties, high thermal conductivity, good thermal reliability and stability. S. Harikrishnan et al. [19] investigated experimentally the solidification and melting characteristics of composite PCMs for building heating application. The increase in thermal conductivity for composite PCMs with mass fraction of $1 \% \mathrm{TiO} 2, \mathrm{ZnO}$ and $\mathrm{CuO}$ nanoparticles has been estimated as $34.85 \%, 46.97 \%$ and $62.12 \%$, respectively while comparing to base material. The experimental results have proved that time savings of composite PCM with $\mathrm{CuO}$ nanoparticles for melting and solidification processes are greater while comparing to the composite PCMs with $\mathrm{TiO} 2, \mathrm{ZnO}$ nanoparticles and base material. Thus, composite PCM with $\mathrm{CuO}$ nanoparticles could be suggested as the potential candidate for building heating applications. In 2015, S. Ramakrishnan et al. [20] introduced a novel paraffin/expanded perlite composite phase change material for prevention of PCM leakage in cementitious composites by impregnating paraffin into hydrophobic coated expanded perlite. The results revealed that incorporation of the developed PCM composite into concrete significantly improved the thermal inertia and thermal energy storage. R. Hossain et al. [21] experimented an energy storage system based on nanoparticle-enhanced phase change material inside porous medium. Numerical and analytical investigations were performed to study the melting process and heat transfer phenomena of nano-PCM inside an enclosure filled with porous medium. Aluminum foam was selected as the porous medium and "Cyclohexane $\mathrm{CuO}$ nanoparticle" was used as the nano-PCM. It is observed that the movement of PCM melting front is more significant under the influence of porous medium than that of nanoparticles. Nano-PCM melts at a faster rate inside the porous medium with lower porosity. For a given porosity of the porous medium, a faster melting rate is observed for nano-PCM with higher volume fraction of nano-particles. From heat transfer rate, it is observed the nano-PCM inside an enclosure with higher porosity requires relatively larger amount of energy to melt when compared with the enclosure having the porous medium with lower porosity.

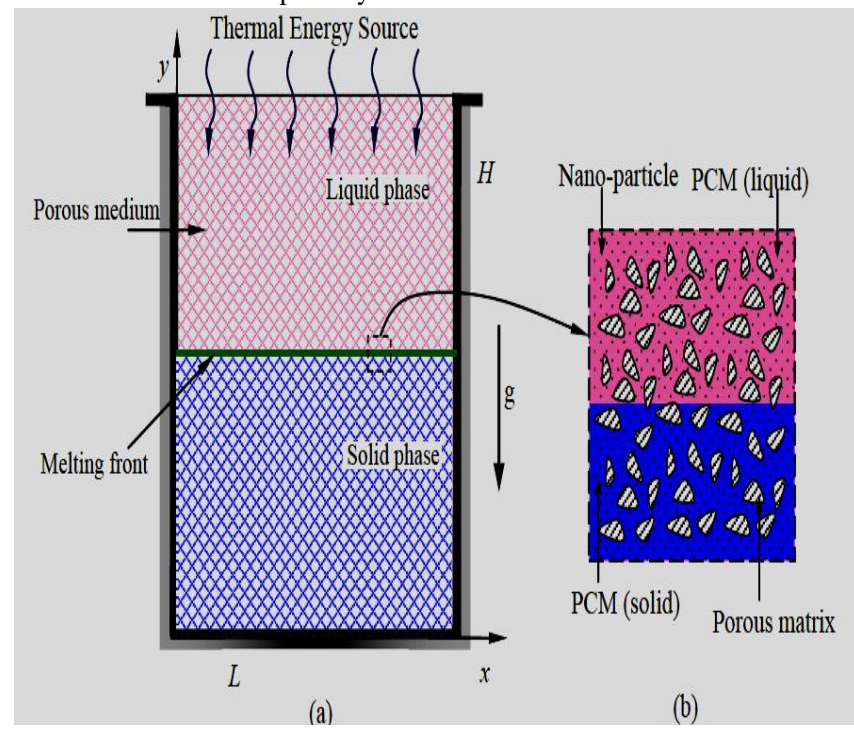

Figure (02): R. Hossain experiments, (a) Schematic diagram of the problem under consideration, (b) a magnified view of a representative control volume 
G. Peiro_et al. [22] performed an Experimental evaluation at pilot plant scale of multiple PCMs (cascaded) against single PCM configuration for thermal energy storage. D-mannitol and hydroquinone were selected as Two PCMs with melting temperatures in a temperature range of $150{ }^{\circ} \mathrm{C}$ to $200{ }^{\circ} \mathrm{C}$. Results showed that the multiple PCMs configuration introduced an effectiveness enhancement of $19.36 \%$ if compared with single PCM configurations. T. Qian et al. [23] examined diatomite as a promising natural candidate as carrier material for low, middle and high temperature phase change material. Polyethylene glycol (PEG), lithium nitrate (LiNO3), and sodium sulfate (Na2SO4) were respectively employed as the low-, middle- and high temperature storage medium. Results indicated that PCMs were well dispersed into diatomite pores and no chemical changes took place during the heating and cooling process. The resulting composites were promising candidates to satisfy the application requirements for different temperature ranges due to their large latent heats and suitable phase change temperatures, as well as the excellent chemical compatibility, improved supercooling extent, and high thermal stability. Zhi Chen and Menghao Qin [24] studied the characterization of composite phase change material with $\mathrm{SiO} 2$ and diatomite finding that the composites had good thermal control. The measurement of thermal properties shows that the $\mathrm{SiO} 2$ can improve the thermal properties. P. Wang et al. [25] studied the thermal energy charging behaviour of a heat exchange device with a zigzag plate configuration containing multi-phase-change-materials. The results show that the use of multiPCMs intensifies the charging process in comparison with the use of a single PCM. Given other conditions, a larger phase change temperature difference between the multi-PCMs gives a more remarkable enhancement of the charging process, and the use of m-PCMs with an unequal mass ratio gives further intensification.

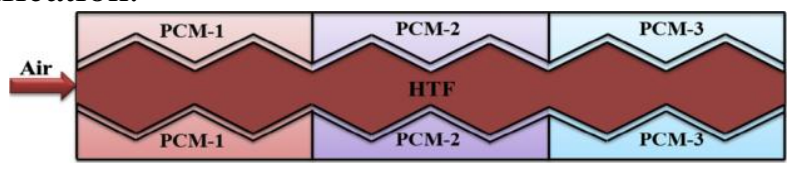

Figure (03): P. Wang experiment, multi-phase change materials in a heat exchange device with a zigzag configuration

A.A. Altohamy et al. [26] studied experimentally the effect of water based Al2O3 nanoparticle PCM on cool storage performance. The results show that there is a significant effect of Nanoparticle concentration on thermal properties of PCM which sequentially reduces complete charging time. In 2016, L.C. Liston et al [27] introduced some mixtures of fatty acid methyl esters as phase change materials for low temperature applications. The aim is to Incorporate phase change materials (PCMs) into concrete pavements has been suggested as a means to improve anti-icing practices by reducing the accumulation of snow and ice providing a solidliquid phase transformation with thermal transition temperature slightly above $0{ }^{\circ} \mathrm{C}$ and with a high enthalpy of fusion. The mixtures candidates and corresponding thermal properties are: (1) methyl laurate $(\mathrm{C} 12)+$ methylmyristate, $x \mathrm{C} 12=0.77$, and (2) methyl laurate + methyl palmitate, $x \mathrm{C} 12=0.86$ with eutectic melting temperatures and latent heats of fusion of $0.21{ }^{\circ} \mathrm{C}$, $2.4^{\circ} \mathrm{C}$, and $174.3 \mathrm{~J} / \mathrm{g}$ and $166.5 \mathrm{~J} / \mathrm{g}$, respectively. Current findings of this study indicate that these binary mixtures have the necessary properties to be a high performance PCM with the potential to reduce the levels of icing on concrete pavements. A. Atal et al. [28] examined the effect of porosity of conducting matrix on a phase change energy storage device. A thermal storage device having a shell and tube arrangement was investigated in this study. The shell contains paraffin wax saturated in aluminum foam. Effect of the porosity of aluminum foams on the thermal 
behavior of the system was investigated by comparing two foams with different porosity (95\% and $77 \%$ ). Both experimental measurements and numerical simulation were performed in this study. In addition, direct observation of the melting/solidification process of the paraffin wax was also made through visualization method. Measurement results were presented and compared with the model predicted solution fields, and good agreements were found. Results showed that the use of conducting matrix with PCM material significantly reduced the time needed for an operating cycle, and that the metal foam with less porosity further shortened the cycle due to higher overall thermal conductivity.
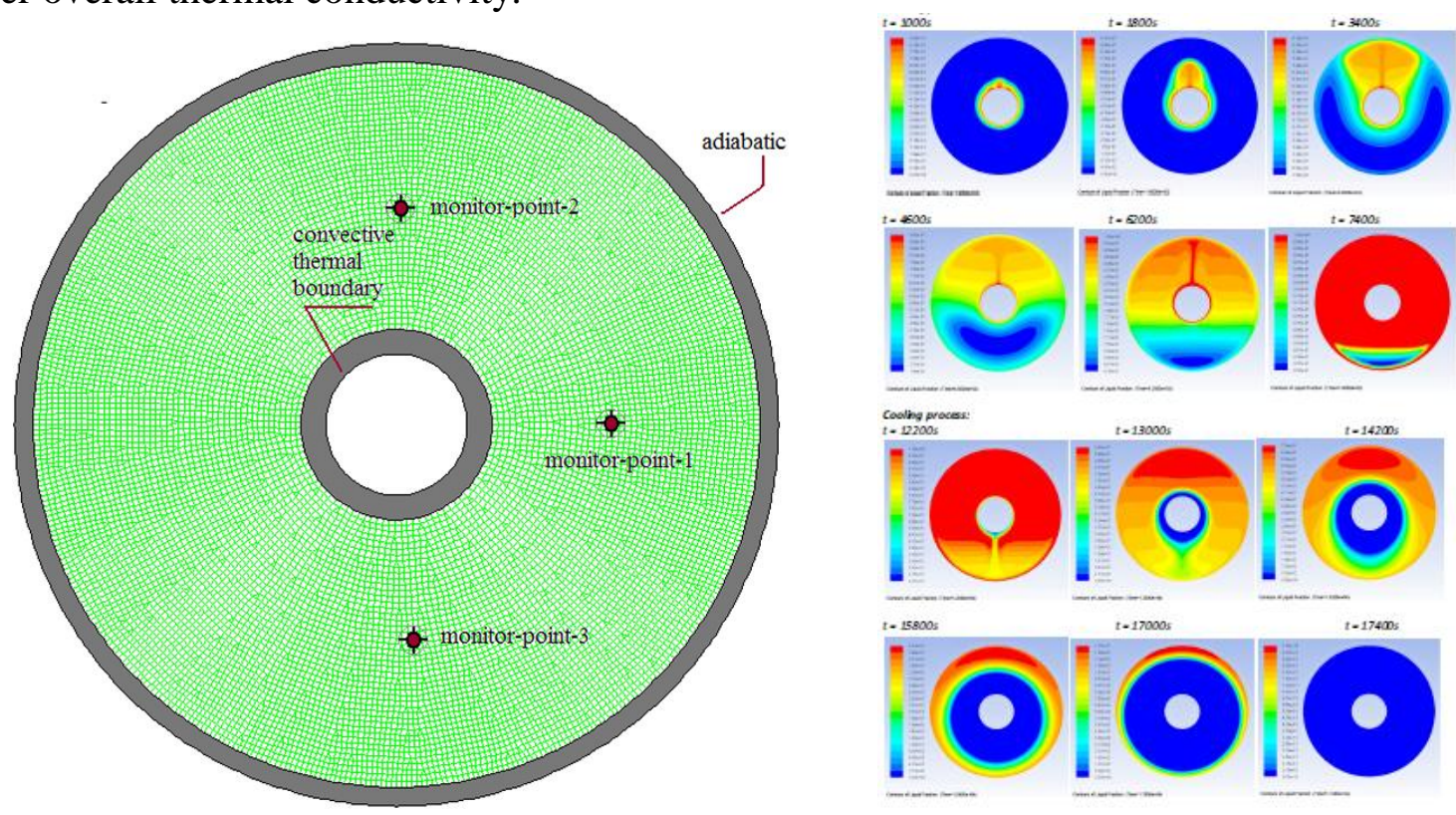

Figure (04): A. Atal trials, computational domain and evolution of solution fields for the paraffin wax configuration

D. Kim et al. [29] presented the structure and thermal properties of octadecane/expanded graphite composites as shape-stabilized phase change materials. Composite properties were strongly dependent on the weight ratio. Analysis showed that OD was adsorbed into the WEPG pores and uniformly covered the surfaces which helped to prevent leakage of melted octadecane during phase change from solid to liquid. The composite with $30 \%$ expanded graphite content showed good thermal properties and shape-stability. Based on these results, it is anticipated that the composites can be used in packaging materials to counteract the negative effects induced by unwanted temperature changes. M. Dannemand et al. [30] studied the solidification behavior and thermal conductivity of bulk sodium acetate trihydrate composites with thickening agents and graphite from the point of view that sodium acetate trihydrate is a promising phase change material for long term storage of solar thermal energy if supercooling is actively utilized. Composite was introduced to enhance heat transfer through the PCM because the contraction and expansion of the salt hydrate during the phase change as well as the relatively low thermal conductivity of the phase change material limits the heat exchange capacity rate to and from the storage. Results indicate that the measured thermal conductivity was up to $0.7 \mathrm{~W} / \mathrm{m} \mathrm{K}$ in solid sodium acetate trihydrate while a composite with sodium acetate trihydrate, thickening agent and 
$5 \%$ graphite flakes had a thermal conductivity of up to $1.1 \mathrm{~W} / \mathrm{m} \mathrm{K}$. Lately, P. Zhang et al. [31] reviewed the composite phase change materials presenting their fabrication, characterization, mathematical modeling and application to performance enhancement. The review considered the recent progresses of the investigations and applications of the composite PCMs with the enhanced performance. The focuses are placed on the composite PCMs fabricated by using the metal foams and carbon materials, which have been proved to be the most promising approaches for thermal conductivity and heat transfer promotion on PCMs concluding that It can be the composite PCMs can effectively improve the performances of LTES systems, which therefore calls for the further investigation in this research field. Finally, the future research topics are suggested. It is found that the heat transfer network is very important in achieving high thermal conductivity of composite PCM fabricated by porous structures. The usage of metal foam and carbon material foam can effectively enhance the thermal conductivities of PCMs on an order of more than ten times or even hundreds times. The assistance of vacuum is an effective way to achieve high impregnation ratio for fabricating composite PCMs with metal/carbon foams. The fabrication methods of carbon material composite PCM include cold compression which compresses carbon materials and PCM solid particles under ambient condition, wet impregnation which soaks carbon materials into liquid PCM to let PCM penetrate into the microstructures or nanostructures, advanced wet impregnation which uses solvent to dissolve PCM to obtain low viscosity solution before impregnation. Vigorous mixing or sonication should be applied during the impregnation process to make the mixture more homogeneous. Vacuum can be also utilized to achieve high loading ratio of PCM in composite. Advanced wet impregnation with the help of vacuum is then believed to be the most effective approach to fabricate composite PCM. Expanded graphite is suggested as an effective thermal conductivity promoter for PCM, and it could be either compressed to graphite foam first or directly mixed and impregnated with liquid PCMs to form composite PCMs. Though carbon nanomaterial and graphene have ultrahigh thermal conductivities, their composite PCMs fabricated by mixing and impregnation method have limited improvement efficacy on thermal conductivity unless higher density composite PCM is made. The higher thermal conductivity of composite PCM leads to a quicker melting and solidification processes. The heat transfer is significantly enhanced in the heat conduction dominated period, though the presence of supporting structure suppresses the natural convection of liquid PCM. The temperature distribution across the PCM is more even. High thermal conductivity is not necessary in some circumstances, then form-stable composite PCM can be fabricated by using polyurethane, silica based materials and so on. The composite PCM could maintain its thermo-physical properties after even thousands heating/cooling cycles.

\section{3- RESEARCHES ABOUT THE CONFIGURATION OF SLAB THAT CONTAINS THE PCM}

Using different configurations of PCM containers is a so attractive field of study that researches investigated through the previous years. In 2011 A. Castell et al. [32] presented an experimental study of a PCM tank for cold storage applications. Two different configurations and different flow rates of the heat transfer fluid were studied. The effectiveness of the PCM storage system was defined as that of a heat exchanger. The results showed that the heat exchange effectiveness of the system did not vary with time, decreased with increasing flow rate and increased with increasing heat transfer area. It was shown that the tube in tank design together with a low temperature PCM is suitable as a thermal storage facility for cold storage. 

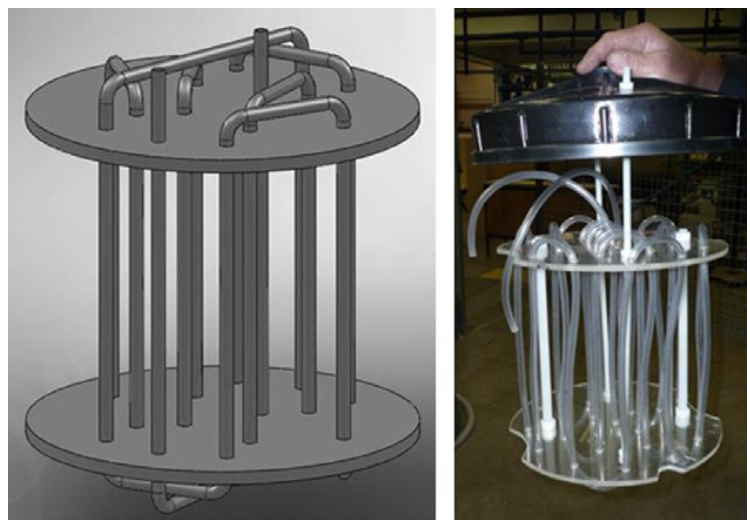

Figure (05): A. Castell, schematic and photo of the experimental PCM tank

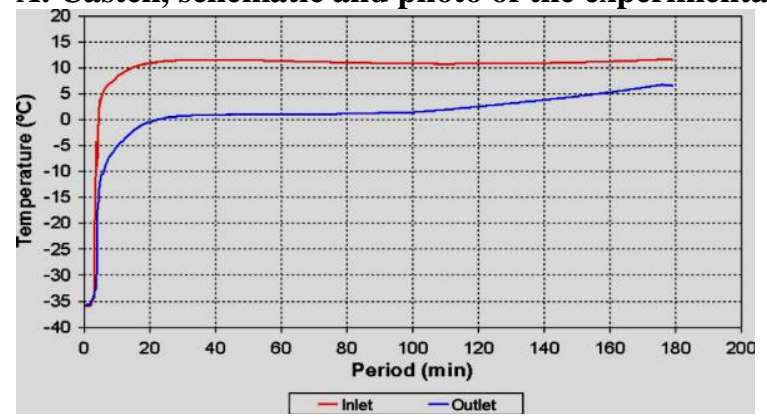

Figure (06): A. Castell, graph showing Inlet and outlet temperatures for the high HTS design for a flow rate of $0.024 \mathrm{~kg} / \mathrm{s}$.

N. Sharifi et al. [33] developed numerical model for simulating the melting of a phase change material (PCM) housed within an internally- finned metal enclosure. The influence of the number of fins, the fin length, thickness, and the hot wall temperature on the melting process is reported. With horizontal fins, rapid melting occurs during the early stages of the phase change, followed by a second, slow melting regime. S.F. Hosseinizadeh et al. [34] performed Experimental and numerical studies on performance of PCM-based heat sink with different configurations of internal fins. The effects of various parameters such as power levels, number of fins, fin height and fin thickness were studied. The results showed that increasing the number of fins and fin height resulted in an appreciable increase in overall thermal performance. Increasing the fin thickness only gave a slight improvement. There was an optimum fin thickness, above which the heat sink performance showed no further improvement. Increasing the power level input, as expected, increased the melting rate of the PCM. 

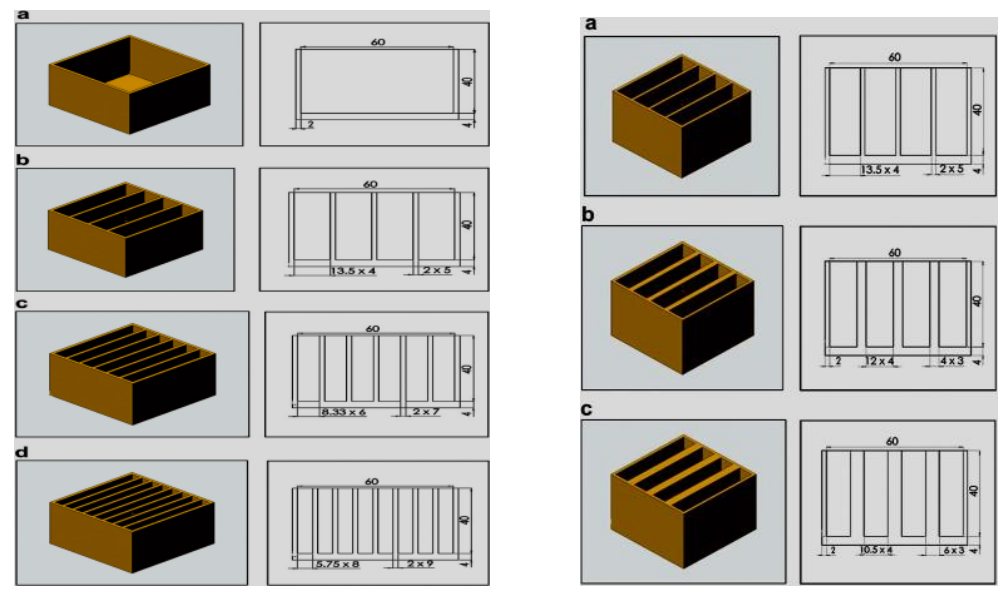

Figure (07): S.F. Hosseinizadeh heat sinks with different number of fins and thickness

C. Balaji et al. [35] tried to find an Optimization of size and shape of composite heat sinks with phase change materials. The base material is aluminum and the phase change material is $n$ Eicosane. Parametric studies were conducted for varying shapes of the fins. To capture the essence of both the time during which the heat sink is used and the time which it takes for dissipating the heat absorbed and be ready for re-use, a term called throughput has been defined. Maximum throughput would indicate maximum amount of heat dissipated over a long period of time when the heat sinks works in cycles. Concluding that for cases where the device being cooled works in cycles of heating and cooling, vertical fins are not the optimum configuration. It is seen that the heat sink with a particular parabolic conducting base material interface, which enhances the overall heat transferred from the device by $122.4 \%$, is a highly acceptable design solution. In 2012, Jingde Zhao et al. [36] simulated numerically the thermal performance of a high aspect ratio thermal energy storage device. The three dimensional numerical study was performed to understand the heat transfer enhancement which results from internal natural convection in a high aspect ratio vertical unit. Octadecane was used as phase change material (PCM) inside TES system, which consisted of six corrugated panels filled with PCM. Each panel had a total of 6 tall cavities filled with PCM, which were exposed to external flow in a concentric TES system. Unlike traditional concentric-type TES devices where heat transfer by conduction is the dominant heat transport mechanism, the high aspect ratio TES configuration used in the study helped promote density-gradient based internal convection mechanism. The results suggest that the high aspect ratio of the TES panels helped induce internal convection near the solid core of octadecane, resulting in a faster melting process.
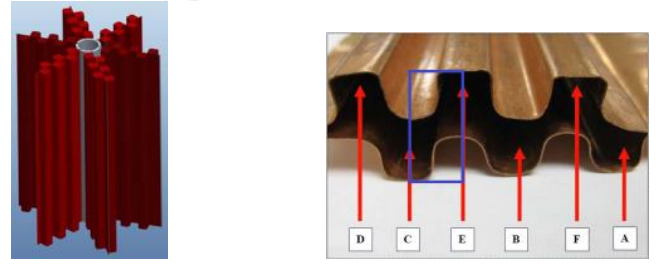

Figure (08): Jingde Zha, 3-D view of TES unit and Top view of a single TES panel In 2013, S. Mat et al. [37] studied the Enhancing of heat transfer for PCM melting in triplex tube with internal-external fins numerically and experimentally. This study numerically investigates 
the melting process in a triplex-tube heat exchanger with phase-change material. Three heating methods were used to melt the PCM from the inside tube, outside tube, and both tubes. Internal, external, and internal- external fin enhancement techniques were studied to improve the heat transfer between the PCM and heat transfer fluid. Enhancement techniques were compared with the inside tube heating, outside tube heating, both tube heating as well as the finned and internally finned tube. The effects of fin length on the enhancement techniques were investigated. Using a triplex-tube heat exchanger with internal- external fins, predicted results indicated that melting time is reduced to $43.3 \%$ in the triplex tube without fins. Experiments were conducted to validate the proposed model. Simulated results correspond with the experimental results.
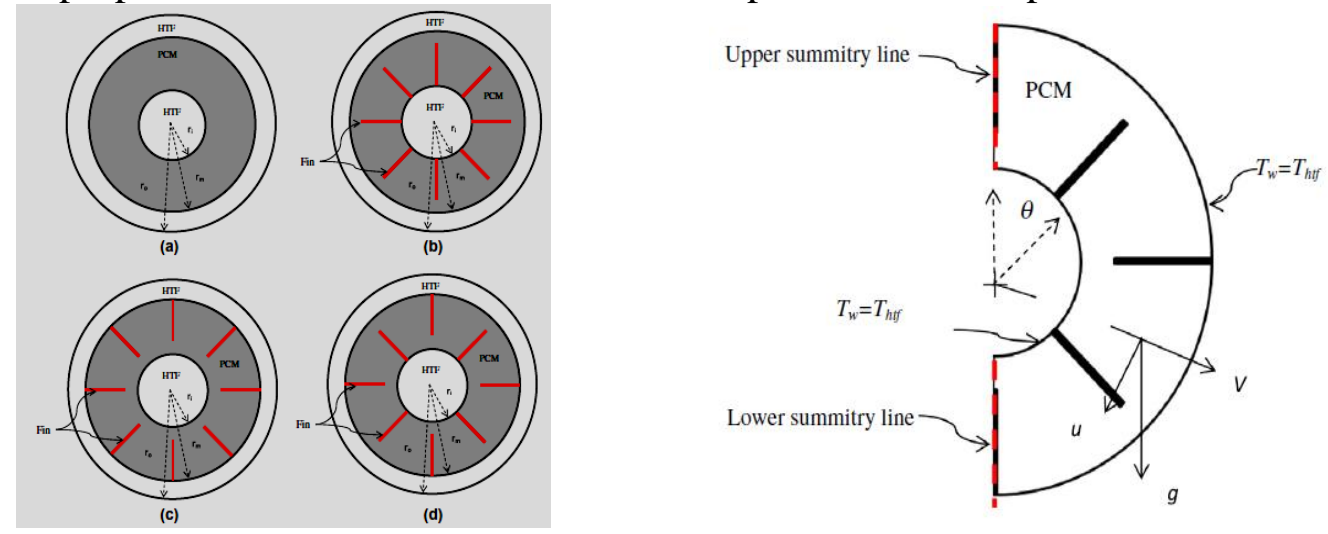

Figure (09): S. Mat, the physical configuration of the TTHX models, (a) TTHX without fin, (b) TTHX with internal fins, (c) TTHX with external fin, (d) TTHX with internal-external fins.

P.P. Levin et al. [38] presented an optimization procedure for the design of a latent heat thermal management system used for cooling an electronic device with transient and high heat generation. The optimized results of this study are presented and discussed, emphasizing the derived optimal PCM percentages, which are an essential parameter in designing an system. These results show that optimal PCM percentages depend on the number and the length of the fins, the heat flux at the interface, and the difference between the critical and liquidus temperatures of the PCM. In 2015, I. Jmal and M. Baccar [39] studied PCM solidification in a finned tube thermal storage numerically. This numerical approach aims to study the impact of natural convection, occurring in the liquid phase, on the solidification time of PCM and the temporal evolution of the solidification front. This paper also investigates the effect of fins number on heat transfer enhancement. The heat transfer enhancement in a PCM-air heat exchanger system using internally and externally finned tubes was numerically investigated. The C18 paraffin as PCM was used and two passages for heating air-flow were considered. In the presence of fins, energy extraction from PCM to airflow occurs at faster rate, which contributes to the reduction of the discharging time and the increase of the outlet air temperature However, for a great number of fins (9 fins), the enhancement of the solidification process is not significant because of the effect of confinement of PCM liquid spaces on the development of thermoconvective flow. During the first $2 \mathrm{~h}$, the increasing temperature of the HTF exceeds $10 \mathrm{C}$. When solidification progresses from the surfaces of the PCM-container and propagates to the central liquid phase, an insulating layer will be created at the vicinity of the container surfaces and the fins, causing a lower heat exchange rate between PCM and air. As the solid PCM layer gets thicker, a progressive regression of the field of movements is induced in the liquid phase, thus 
leading to the inhibition of heat extraction process. After about $7 \mathrm{~h}, 60 \%$ of the PCM became solid, and heat transfer was almost dominated by conduction mechanism.
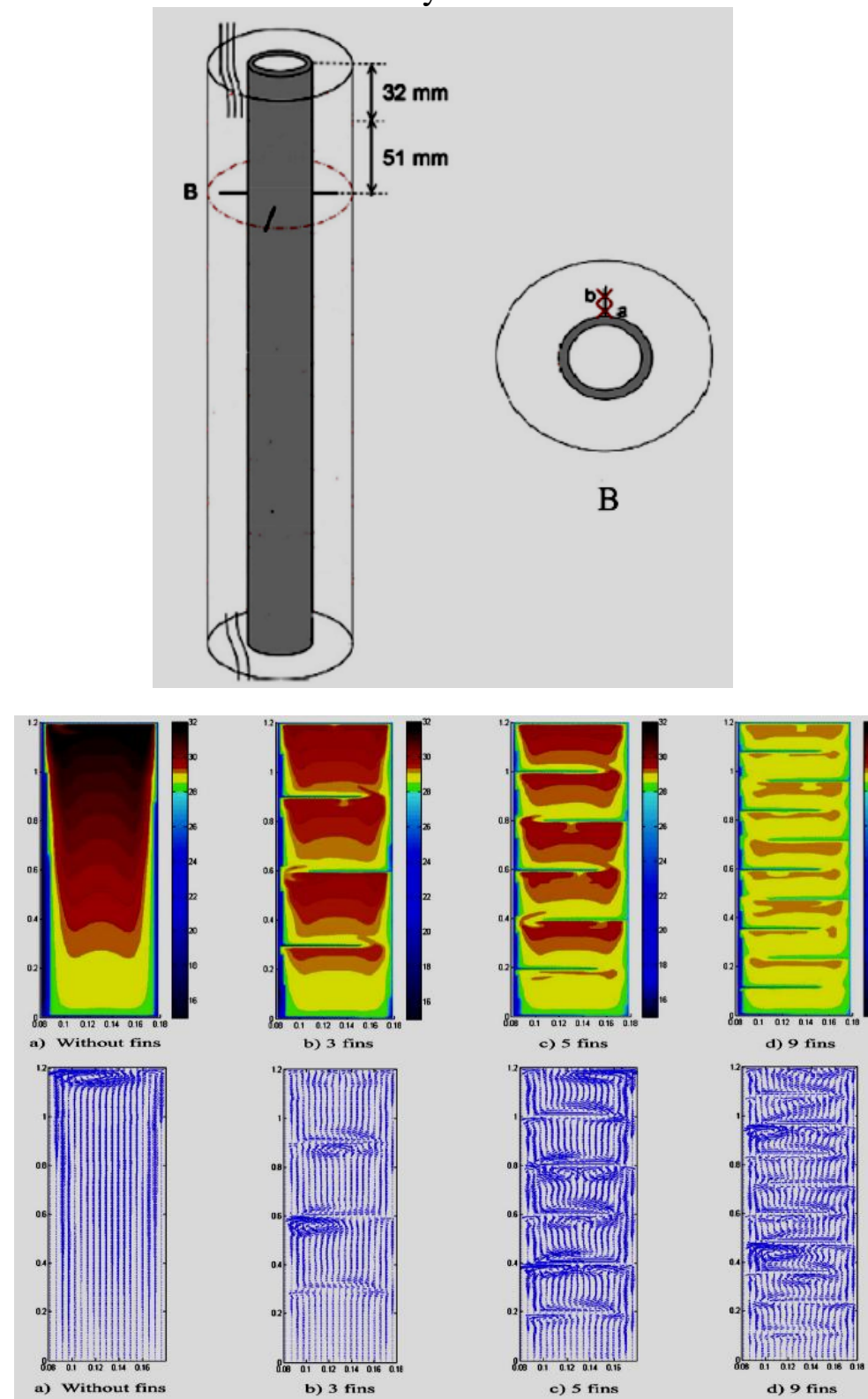

Figure (10): I. Jmal and M. Baccar, scheme of the experimental loop and thermocouple positions in the test section and showing Temperature fields and corresponding velocity vectors at $t 1 / 4 \mathrm{~h}$ for different fin numbers $(0,3,5$ and 9 fins)

L. Yang et al. [40] analyzed numerically the performance of naphthalene phase change thermal storage system in aluminum plate-fin unit. The prediction of time, temperature and liquid fraction during the melting and solidification process in the latent heat storage of phase changed materials (PCM) was simulated. The results showed that the thermal storage with internal fins gives better thermal performance compared to one without internal fins. Fins in the aluminum plate-fin thermal storage have been accelerated the heat transfer rate. Therefore, fins improve the energy storage and release performance. Also, the smaller fin pitches, the shorter melting/solidification 
period. This is due mainly to the smaller PCM volume and larger heat transfer surface in heat storage unit. While increasing the fin length will yield no further improvement in thermal performance. In 2016, P. Wang et al. [41] investigated numerically the PCM melting process in a sleeve tube with internal fins. In this work, a detailed numerical study is carried out to analyze the impact of fin geometry (including fin-length, fin-ratio and the angle between neighbor fins) on PCM melting process. Results shows that the heat transfer can be enhanced by adding halfscale fins in the sleeve-tube thermal energy unit and the fin-ratio has effect to shorten melting time of PCM, but the effect of reducing fin-ratio to speed melting process is not remarkable.

\section{4- RESEARCHES INTRODUCING ENCAPSULATION TECHNOLOGY OF PCM} TO ALLOW BETTER HEAT TRANSFER

Researchers worked on studying encapsulation of PCM in order to avoid leakage when in liquid state as well as allowing larger surface area for heat transfer and protection in handling hazardous materials. In 2011, V.V. Tyagi et al. [42] published a review discussing the Development of phase change materials based microencapsulated technology for buildings. Microencapsulation is the process by which individual particles or droplets of solid or liquid material (the core) are surrounded or coated with a continuous film of polymeric material (the shell) to produce capsules in the micrometer to millimeter range, known as microcapsules. From the aspect that the microencapsulation is one of the well-known and advanced technologies for better utilization of PCMs with building parts, such as, wall, roof and floor besides, within the building materials. One problem to solve in some PCM applications is the liquid migration, using some kinds of packing. Microcapsules consist of little containers, which pack a core material with a hard shell. Microencapsulating PCM brings some more important advantages like that microcapsules can handle phase change materials as core, as far as, they tolerate volume changes. The description of microcapsules depends mainly on the core material and the deposition process of the shell 1. Mononuclear (core-shell) microcapsules contain the shell around the core.

2. Polynuclear capsules have many cores enclosed within the shell.

3. Matrix encapsulation in which the core material is distributed homogeneously into the shell material.
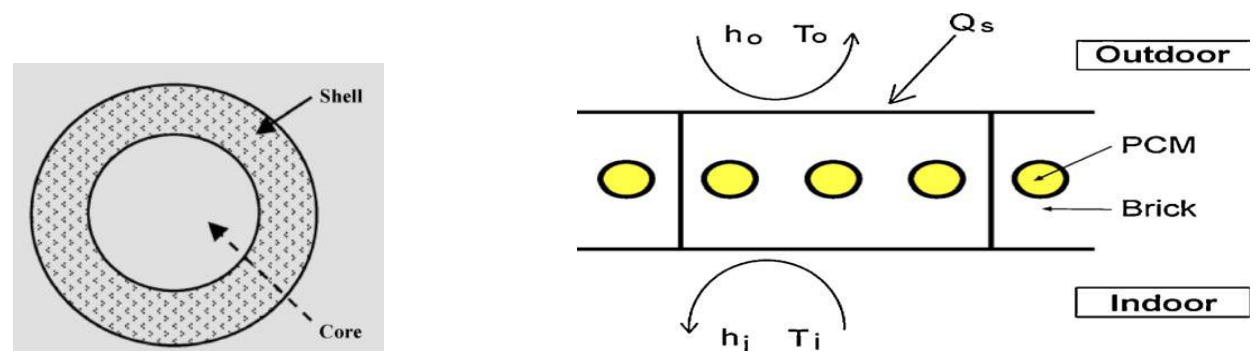

Figure (11): V.V. Tyagi, description of microcapsule and schematic representation of the Brick-PCM system showing boundary conditions. 


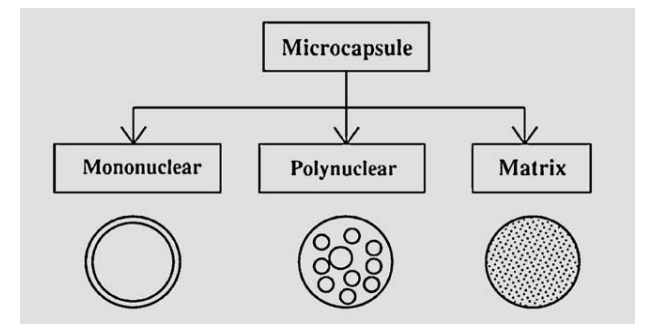

Figure (12): V.V. Tyagi, types of microcapsule.

Several physical and chemical methods have been developed for production of microcapsules. The most often used microencapsulation methods are:

Physical methods:

(i) Pan coating

(ii) Air-suspension coating

(iii) Centrifugal extrusion

(iv) Vibrational Nozzle

(v) Spray drying

Chemical methods:

(i) Interfacial polymerization

(ii) In situ polymerization

(iii) Matrix polymerization

In 2012, P.B. Salunkhe and P.S. Shembekar [43] also reviewed the effect of phase change material encapsulation on the thermal performance of a heat storage system. The key encapsulation parameters, namely, encapsulation sizes, shell thickness, shell material and encapsulation geometry have been investigated thoroughly. It was observed that the core-tocoating ratio plays an important role in deciding the thermal and structural stability of the encapsulated PCM. An increased core-to-coating ratio results in a weak encapsulation, whereas, the amount of PCM and hence the heat storage capacity decreases with a decreased core-tocoating ratio. Types of PCM encapsulation based on size Based on size, encapsulated PCM can be classified as follows:

i) Macro (above $1 \mathrm{~mm}$ ),

ii) Micro $(0-1000 \mathrm{~mm})$ and

iii) Nano (0-1000 nm) encapsulated PCM.

Thermal conductivity of shell material found to have a significant influence on the heat exchange between the PCM and heat transfer fluid. So that, a higher thermal conductivity of shell material, a lower shell size and high temperature of HTF results in rapid melting of the encapsulated PCM. In 2014, A. Jamekhorshid et al. [44] reviewed microencapsulation methods of phase change materials (PCMs) as a thermal energy storage (TES) medium. In this paper, microencapsulation methods are perused and classified into three categories, i.e. physical, physic-chemical, and chemical methods. The most often used microencapsulation methods are as follows:

Physical methods:

i- Pan coating,

ii- Air-suspension coating,

iii- Centrifugal extrusion, 
iv- Vibrational nozzle,

v- Spray drying,

vi- Solvent evaporation.

Physic-chemical methods:

i- Ionic gelation,

ii- Coacervation,

iii- Sol-gel

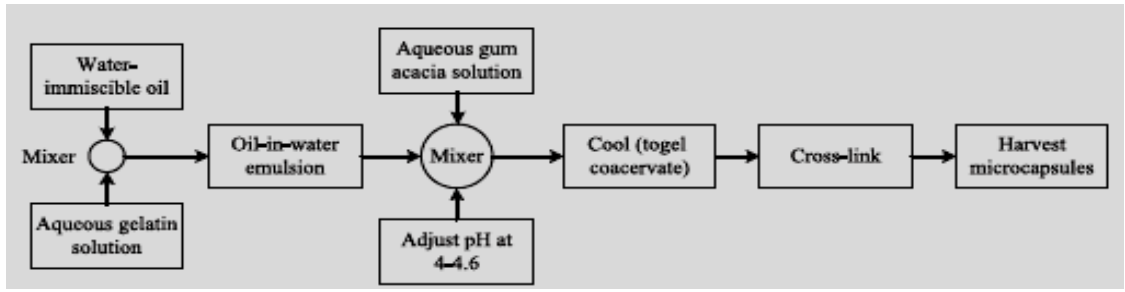

Figure (13): A. Jamekhorshid, flow diagram of a typical complex coacervation encapsulation process

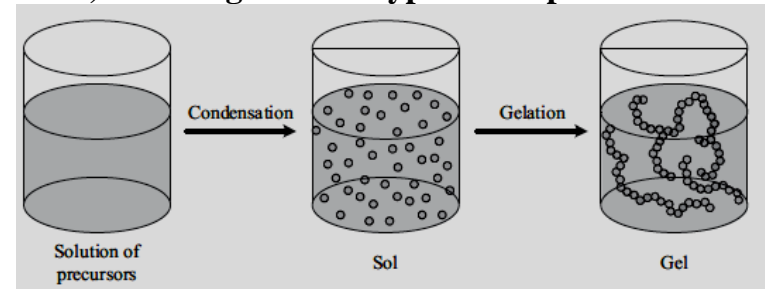

Chemical methods:

Figure (14): A. Jamekhorshid, scheme of a sol-gel process

i. Interfacial polymerization

ii. Suspension polymerization

iii. Emulsion polymerization

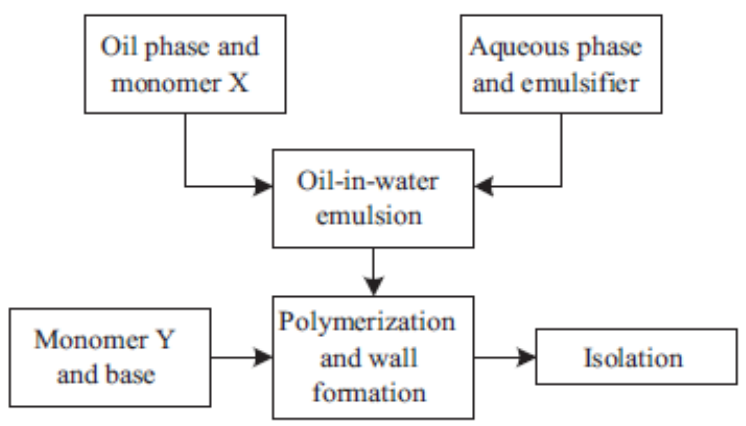

Fig. (15): A. Jamekhorshid, flow diagram of a typical interfacial polymerization encapsulation process Among all the microencapsulation methods, the most common methods described in the literature for the production of microencapsulated phase change materials (MEPCMs) are interfacial polymerization, suspension polymerization, coacervation, emulsion polymerization, and spray drying. Concluding Summary

The accumulated works performed by researchers lead to introducing some ideas that can magnify the thermal performance of PCMs includes the following:

1- Some promising substances are very promising to be used as a PCM such as: 
REVIEW ON ENHANCING TECHNOLOGIES OF HEAT TRANSFER THROUGH PHASE CHANGE MATERIALS

- Pure inorganic salts like (low cost chlorides, nitrates and carbonates)

- $\quad$ Salt eutectics like (KNO3-LiNO3 and KNO3-NaNO3-LiNO3)

- Liquid metals like (Gallium)

- Esters

- Nanofluids (A12O3/water, $\mathrm{CuO} /$ water and alumina-water)

- Polyols (sugar alcohol like polyethelene glycol, erythritol and xylitol)

2- Some composite mixtures are very promising to be used as a PCM such as:

- Fatty acid esters with building materials like concrete pavements, cement or gypsum

- Fatty acid esters with isolating materials like perlite to keep satisfying room temperature for long time.

- PCMs mixed with good conducting materials like graphite and aluminum

- PCMs mixed with stable materials like Diatomite and silica

- PCMs mixed with Nano particles of TiO2, $\mathrm{ZnO}, \mathrm{CuO}$ and Silver-Titania

3- Configuration of PCM container can be modified to achieve higher heat transfer rates enhancing the PCM thermal performance as follows:

- Adding internal fins. Experiments advise to focus on increasing the number and height of fins more than focusing of fins thickness.

- Experiments on adding external fins advise to focus on increasing the number and height of fins more than focusing of fins thickness.

- Increasing the aspect ratio of the PCM vertical container to induce natural convection.

4- Encapsulating technology in order to avoid leakage when in liquid state as well as allowing larger surface area for heat transfer and protection in handling hazardous materials

\section{REFERENCES}

1- Ming Liu, Wasim Saman, Frank Bruno. Review on storage materials and thermal performance enhancement techniques for high temperature phase change thermal storage systems. Renewable and Sustainable Energy Reviews 16 (2012) 2118- 2132

2- Karim Menoufi, Albert Castell, Mohammed M Farid, Dieter Boer, Luisa F Cabeza. Life Cycle Assessment of experimental cubicles including PCM manufactured from natural

3- Haoshan Ge, Haiyan Li, Shengfu Mei, Jing Liu. Low melting point liquid metal as a new class of phase change material: An emerging frontier in energy area. Renewable and Sustainable Energy Reviews 21 (2013) 331-346

4- Antoni Gil, Camila Barreneche, Pere Moreno, Cristian Solé, A Inés Fernández, Luisa F Cabeza. Thermal behaviour of D-mannitol when used as PCM: Comparison of results obtained by DSC and in a thermal energy storage unit at pilot plant scale. Applied Energy 111 (2013) 1107-1113

5- F Roget, C Favotto, J Rogez. Study of the KNO3-LiNO3 and KNO3-NaNO3-LiNO3 eutectics as phase change materials for thermal storage in a low-temperature solar power plant. Solar Energy 95 (2013) 155-169

6- A Sivakumar, N Alagumurthi, $\mathrm{T}$ Senthilvelan. Experimental investigation of forced convective heat transfer performance in nanofluids of $\mathrm{Al} 2 \mathrm{O} 3 /$ water and $\mathrm{CuO} /$ water in a serpentine shaped micro channel heat sink. Heat Mass Transfer DOI 10.1007/s00231-015$1649-5$

7- $\quad$ M M MacDevette, T G Myers. Nanofluids: An innovative phase change material for cold storage systems. International Journal of Heat and Mass Transfer 92 (2016) 550-557 
REVIEW ON ENHANCING TECHNOLOGIES OF HEAT TRANSFER THROUGH PHASE CHANGE MATERIALS

8- $\quad$ Saman Nimali Gunasekara, Ruijun Pan, Justin Ningwei Chiu, Viktoria Martin. Polyols as phase change materials for surplus thermal energy storage. Applied Energy 162 (2016) 1439-1452

9- Ali Karaipekli, Ahmet Sarı. Preparation and characterization of fatty acid ester/building material composites for thermal energy storage in buildings. Energy and Buildings 43 (2011) 1952-1959

10- Yi Wanga, Tian Dong Xi, Han Zhengc, Hui Xia Feng. Stearic acid/silica fume composite as form-stable phase change material for thermal energy storage. Energy and Buildings 43 (2011) 2365-2370

11- Yong Qian, Ping Wei, Pingkai Jiang, Jiping Liu. Preparation of halogen-free flame retardant hybrid paraffin composites as thermal energy storage materials by in-situ sol-gel process. Solar Energy Materials \& Solar Cells 107 (2012) 13-19

12- S F Hosseinizadeh, A A Rabienataj Darzi, F L Tan. Numerical investigations of unconstrained melting of nano-enhanced phase change material (NEPCM) inside a spherical container. International Journal of Thermal Sciences 51 (2012) 77e83.

13- Mohammad Mehrali, Sara Tahan Latibari, Mehdi Mehrali, Teuku Meurah Indra Mahlia, Hendrik Simon, Cornelis Metselaar. Preparation and properties of highly conductive palmitic acid/ graphene oxide composites as thermal energy storage materials. Energy 58 (2013) 628e634

14- Su-Gwang Jeong, Jisoo Jeon, Jeong-Hun Lee, Sumin Kim. Optimal preparation of PCM/diatomite composites for enhancing thermal properties. International Journal of Heat and Mass Transfer 62 (2013) 711-717

15- X L Wang, X Q Zhai, T Wang, H X Wang, Y L Yin. Performance of the capric and lauric acid mixture with additives as cold storage materials for high temperature cooling application. Applied Thermal Engineering 58 (2013) 252e260

16- Min Li. A nano-graphite/paraffin phase change material with high thermal Conductivity. Applied Energy 106 (2013) 25-30

17- R Parameshwaran, K Deepak, R Saravanan, S Kalaiselvam. Preparation, thermal and rheological properties of hybrid nanocomposite phase change material for thermal energy storage. Applied Energy 115 (2014) 320-330

18- Nan Zhang, Yanping Yuan, Yaguang Yuan, Tianyu Li, Xiaoling Cao. Lauric-palmiticstearic acid/expanded perlite composite as form-stable phase change material: Preparation and thermal properties. Energy and Buildings 82 (2014) 505-511

19- S Harikrishnan, M Deenadhayalan, S Kalaiselvam. Experimental investigation of solidification and melting characteristics of composite PCMs for building heating application. Energy Conversion and Management 86 (2014) 864-872

20- Sayanthan Ramakrishnan, Jay Sanjayan, Xiaoming Wang, Morshed Alam, John Wilson. A novel paraffin/expanded perlite composite phase change material for prevention of PCM leakage in cementitious composites. Applied Energy 157 (2015) 85-94

21- Rakib Hossain, Shohel Mahmud, Animesh Dutta, Ioan Pop. Energy storage system based on nanoparticle-enhanced phase change material inside porous medium. International Journal of Thermal Sciences 91 (2015) 49e58

22- Gerard Peir, Jaume Gasia, Laia Mir, Luisa F Cabeza. Experimental evaluation at pilot plant scale of multiple PCMs (cascaded) vs. single PCM configuration for thermal energy storage. Renewable Energy 83 (2015) 729e736 
23- Tingting Qian, Jinhong Li, Xin Min, Yong Deng, Weimin Guan, Lei Ning. Diatomite: A promising natural candidate as carrier material for low, middle and high temperature phase change material. Energy Conversion and Management 98 (2015) 34-45

24- Zhi Chena, Menghao Qin. Synthesis and characterization of composite phase change material (CPCM) with $\mathrm{SiO} 2$ and diatomite as endothermal-hygroscopic material. Energy Procedia 78 ( 2015 ) $201-206$

25- Peilun Wang, Xiang Wang, Yun Huang, Chuan Li, Zhijian Peng, Yulong Ding. Thermal energy charging behaviour of a heat exchange device with a zigzag plate configuration containing multi-phase-change-materials (m-PCMs). Applied Energy 142 (2015) 328-336

26- Ahmed A Altohamy, M F Abd Rabbo, R Y Sakr, Ahmed A A Attia. Effect of water based A12O3 nanoparticle PCM on cool storage performance. Applied Thermal Engineering 84 (2015) 331e338

27- Leah C Liston, Yaghoob Farnam, Matthew Krafcik, Jason Weiss, Kendra Erk, Bernard Y Tao. Binary mixtures of fatty acid methyl esters as phase change materials for low temperature applications. Applied Thermal Engineering 96 (2016) 501-507

28- Aditya Atal, Yuping Wang, Mayur Harsha, Subrata Sengupta. Effect of porosity of conducting matrix on a phase change energy storage device. International Journal of Heat and Mass Transfer 93 (2016) 9-16

29- Dowan Kim, Jeyoung Jung, Youngno Kim, Miji Lee, Jongchul Seo, Sher Bahadar Khan. Structure and thermal properties of octadecane/expanded graphite composites as shapestabilized phase change materials. International Journal of Heat and Mass Transfer 95 (2016) 735-741

30- Mark Dannemand, Jakob Berg Johansen, Simon Furbo. Solidification behavior and thermal conductivity of bulk sodium acetate trihydrate composites with thickening agents and graphite. Solar Energy Materials \& Solar Cells 145 (2016) 287-295

31- $\mathrm{P}$ Zhang, X Xiao, and Z W Ma. A review of the composite phase change materials: Fabrication, characterization, mathematical modeling and application to performance enhancement. Applied Energy 165 (2016) 472-510

32- A Castell, M Belusko, F Bruno, L F Cabeza. Maximisation of heat transfer in a coil in tank PCM cold storage system. Applied Energy 88 (2011) 4120-4127

33- Nourouddin Sharifi, Theodore L Bergman, Amir Faghri. Enhancement of PCM melting in enclosures with horizontally-finned internal surfaces. International Journal of Heat and Mass Transfer 54 (2011) 4182-4192

34- S F Hosseinizadeh, F L Tan, S M Moosania. Experimental and numerical studies on performance of PCM-based heat sink with different configurations of internal fins. Applied Thermal Engineering 31 (2011) 3827e3838

35- C Balaji, Praneet Mungara, Parw Sharma. Optimization of size and shape of composite heat sinks with phase change materials. Heat Mass Transfer (2011) 47:597-608 DOI 10.1007/s00231-010-0752-X

36- Jingde Zhao, Ehsan M Languri, Jorge L Alvarado, Chao Wang. NUMERICAL SIMULATION OF THERMAL PERFORMANCE OF A HIGH ASPECT RATIO THERMAL ENERGY STORAGE DEVICE. Proceedings of the ASME 2012-89482 International Mechanical Engineering Congress \& Exposition IMECE2012 November 9-15, 2012, Houston, Texas, USA 
37- Sohif Mat, Abduljalil A Al-Abidi, K Sopian, M Y Sulaiman, Abdulrahman Th Mohammad. Enhance heat transfer for PCM melting in triplex tube with internal-external fins. Energy Conversion and Management 74 (2013) 223-236

38- Peleg P Levin, Avraham Shitzer, Gad Hetsroni. Numerical optimization of a PCM-based heat sink with internal fins. International Journal of Heat and Mass Transfer 61 (2013) 638645

39- Imen Jmal, Mounir Baccar. Numerical study of PCM solidification in a finned tube thermal storage including natural convection. Applied Thermal Engineering 84 (2015) 320e330

40- Lin Yang, Hao Peng, Xiang Ling, Huihua Dong. Numerical analysis on performance of naphthalene phase change thermal storage system in aluminum plate-fin unit. Heat Mass Transfer (2015) 51:195-207 DOI 10.1007/s00231-014-1400-7

41- Peilun Wang, Hua Yao, Zhipeng Lan, Zhijian Peng, Yun Huang, Yulong Ding. Numerical investigation of PCM melting process in sleeve tube with internal fins. Energy Conversion and Management 110 (2016) 428-435 